\title{
Hedonics of Food Consumption: Are Food 'Liking' and 'Wanting' Viable Targets for Appetite Control in the Obese?
}

\author{
Graham Finlayson • Michelle Dalton
}

Published online: 10 January 2012

(C) Springer Science+Business Media, LLC 2012

\begin{abstract}
In an environment where energy-dense and highly palatable foods are ubiquitous, it seems plausible that the hedonic system of appetite control will play a primary role in eating behavior - undermining homeostatic processes and driving consumption beyond energy requirements. A relevant issue in the hedonics of food consumption is the distinction between "liking" and "wanting" components of food reward. Separate neural pathways exist to mediate these processes, and experimental behavioral methods have been developed to distinguish and to measure them separately in humans. We examine the evidence that "liking" and "wanting" are involved in weight gain, obesity, and certain forms of disordered eating. Then it is questioned whether "liking" and "wanting" are involved in "food addiction." We conclude that elevated "liking" and "wanting" are psychological markers of a susceptible phenotype for overconsumption. These processes contribute to what can be termed "hedonically driven eating" and represent viable targets for appetite control.
\end{abstract}

Keywords "Liking" . "Wanting" · Food hedonics · Reward . Hedonic hotspots · Incentive salience · Appetite control .

Weight gain · Obesity $\cdot$ Binge eating $\cdot$ Restrained eating $\cdot$ Food addiction $\cdot$ Psychology $\cdot$ Food consumption

\section{Introduction}

Overweight and obesity are a global problem. In 2008, an estimated 1.46 billion adults were overweight worldwide, and of these 502 million were obese [1]. This striking increase over a relatively short period of time has coincided

G. Finlayson $(\bowtie) \cdot$ M. Dalton

Institute of Psychological Sciences, University of Leeds,

Leeds, West Yorkshire LS2 9JT, United Kingdom

e-mail: g.s.finlayson@leeds.ac.uk with changes to the environment, in which energy-dense and palatable foods are increasingly available and served in larger portions [2]. Meanwhile, advances in technology have meant that populations are increasingly sedentary [3]. Considering these developments, it can be agreed that the environment in which many people now live is "obesogenic," with few barriers to prevent repeated overconsumption of hyper-palatable, energy-dense foods that are high in fat and sugar. However, it is also apparent that not all individuals have the same vulnerability to the availability of highly rewarding food or the pervasive inducements to consume them. Individual differences in the hedonic aspects of food intake can be linked to genetic, physiologic, metabolic, and psychological factors, all of which can be viewed as part of an integrated psychobiological system (Fig. 1). It is generally agreed that the psychobiological system comprises homeostatic as well as hedonic features of appetite that work in parallel to influence food intake $[4,5]$. The homeostatic appetite system coordinated by the hypothalamus responds to internal signals of energy requirement and modulates the motivation to eat via sensations of hunger and fullness. The hedonic appetite system coordinated by the brain's reward circuitry, predominantly underpinned by dopamine and opioid transmission in the striatum [4], responds to sensory properties and thoughts about food that foster "liking" and "wanting." Both systems operate to maintain appropriate levels of energy based on the monitoring of short-term "episodic" and long-term "tonic" energy requirements [6]. Therefore, it is to be expected that there will be considerable overlap between the homeostatic and hedonic domains in the control of food intake [7]. Serious malfunctions in the psychobiological regulation of energy such as congenital leptin deficiency can result in the development of obesity [8] but such conditions are relatively rare. In an environment in which energy-dense and highly palatable foods are ubiquitous, 


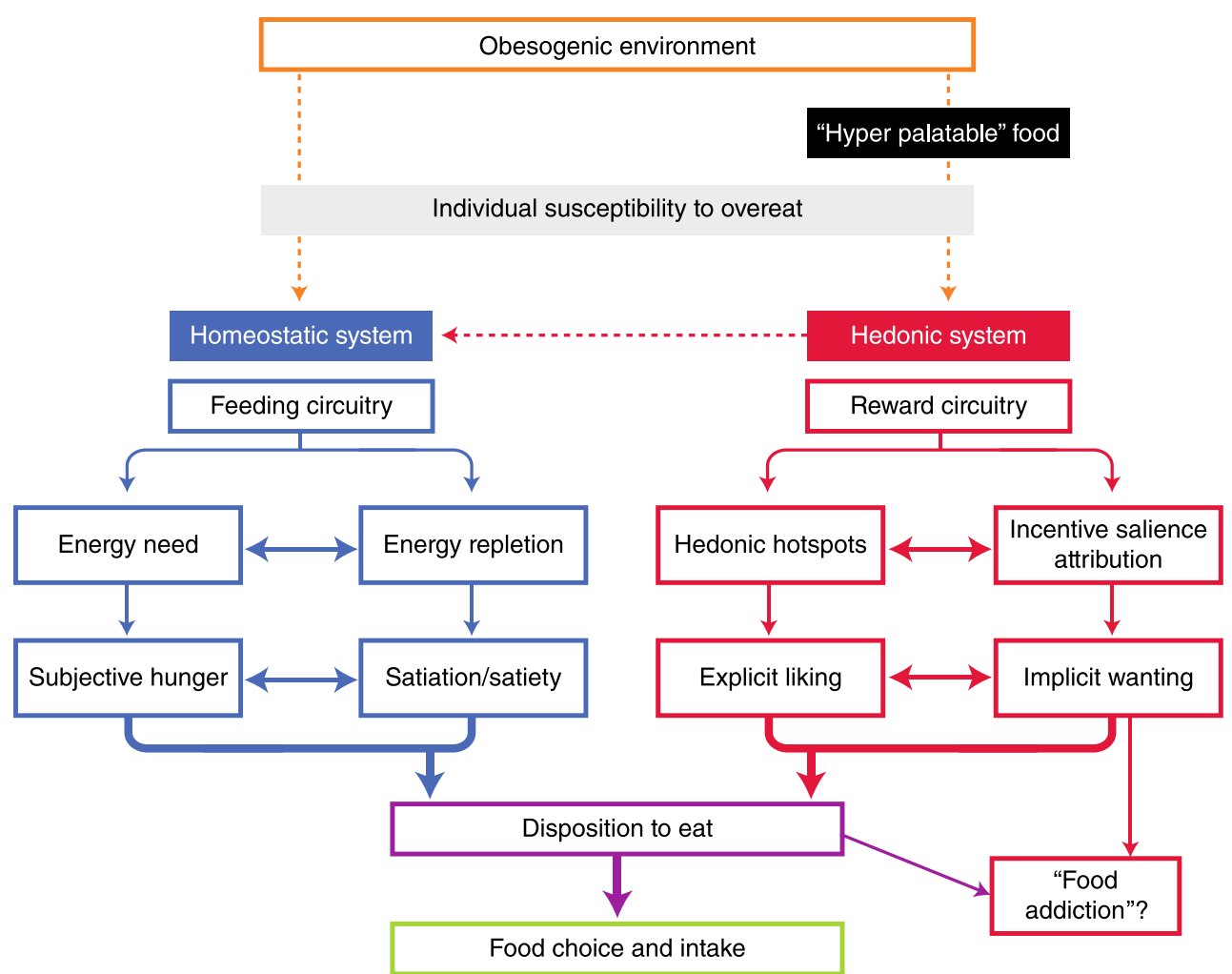

Fig. 1 Illustrates how hedonic and homeostatic systems operate in an "obesogenic environment" to drive overconsumption. Highly palatable foods (often high in fat and sugar) interact with individual susceptibility to overeat. The resulting activation of the hedonic system is marked by an elevation in "liking" and "wanting" for food underpinned by opioid and dopamine transmission in the brain's reward circuitry. The hedonic system amplifies the motivation to eat and weakens inhibition of this drive by

it seems plausible that processes in the hedonic system of appetite control, independent from their involvement in normal homeostatic regulation, play a more important role in food intake behavior - capable of amplifying the motivation to eat, weakening inhibitory signals of satiety, and driving consumption outside of energy needs. Recently, a good deal of progress has been achieved in understanding the hedonic response to food and how separate psychological components of food "liking" and "wanting" feature in overconsumption and obesity. The aims of this review are to present current thinking on the neurobiology of food hedonics and specifically the emergence of "liking" and "wanting" as separable and measureable components of food reward; to examine the role of "liking" and "wanting" in forms of disordered eating behavior; and to discuss their involvement in the concept of "food addiction."

\section{Current Thinking on the Neurobiology of Food Hedonics}

For more than a decade, Berridge's $[9,10]$ influential theory of reward has provided a useful framework for investigating satiety, therefore operating outside of the normal homeostatic regulation of food intake. Adaptations in homeostatic and hedonic processes characterize individual differences in the disposition to eat such as the tendency to be restrained or binge eat. Finally, chronic overconsumption of high-fat, highreward food may compound pathologic eating patterns such as those seen in severe binge eating disorder

the role of hedonics (processes of, or relating to, pleasure and reward) in human appetitive behavior. The theory posits that reward is not a unitary process but encompasses both an affective pleasure component and a non-affective motivational component, termed "liking" and "wanting," respectively. "Liking" underpins the subjective pleasure elicited by food. Food "liking" is generated by the release of endogenous opioids in response to food acting on localized clusters of neurons termed hedonic "hotspots" [11]. To date, hedonic hotspots have been identified in the nucleus accumbens, the ventral pallidum, and the brainstem. These hotspots are thought to interact, creating a distributed network of "liking" sites from the limbic forebrain to the brainstem [12, 13••, 14]. "Liking" reactions are mediated predominantly by opioidergic, but also to some extent by endocannabinoid and GABAergic signals [13••]. Research in non-human animals has shown that microinjections of the $\mu$-opioid agonist, DAMGO, increased "liking" for sucrose in both behavioral (increased number of positive affective taste reactions) and neural (firing signals of ventral pallidum neurons) responses [15]. "Wanting" is the motivational component of reward, referred to as a process of "incentive salience attribution" to 
rewards and their associated cues in the environment [10]. Food "wanting" arises through the release of dopamine in the mesocorticolimbic pathway prior to and during contact with food. Importantly, it is through the action of dopamine and not opioids that foods are given their motivational appeal; and opioids in the absence of dopamine are sufficient to elicit affective responses [10]. Although food "liking" has been described as a kind of "pleasure gloss," actively painted onto the sensory perception of food [16], "wanting" accounts for the expression of behavior that does not normally have a verbal or explicit explanation (eg, "I just had to have it! I don't know why."). "Liking" and "wanting" have distinguishable substrates in brain and although hedonic aspects of food intake are typically a combination of both "liking" and "wanting," by measuring these components separately in behavior, we may learn under which circumstances they differ by degree or even dissociate and how this might determine an individual's propensity to overconsume and gain weight [7].

\section{'Liking' and 'Wanting' as Psychological Components of Reward}

Although "liking" and "wanting" as dissociable components of reward have acquired some validity from neurobiological processes encoded in the brain, the terms "liking" and "wanting" are also derived from the semantics of a shared language that describes human activities. "Liking" and "wanting" have separate meanings in the language with different motivational connotations. Food "liking" and "wanting," expressed to denote specific neural processes of reward, are often discussed in relation to subjective states or feelings that correspond to the ordinary understanding of these terms (and their synonyms) in the context of human appetite behavior, such as food choice and food intake. Although use of the terms in human behavior has a certain overlap with their use by animal theorists, the concepts do not share an identity and it is important to point out that their validity does not have to be sought through shared theoretical mechanisms. "Wanting" may refer to subjective states of desire, craving, or literally to feel a "lack [of] something desirable or necessary (especially a quality or attribute)" [17], whereas "liking" is usually understood as the perceived impact of a food or its sensory properties on subjective affect or some judgment of the pleasure it elicits. Although it is assumed that the intensity and valence of our subjective experience of reward are reflective of changes in these core processes, the link between subjective sensations and objective neurochemical events is far from understood. There are instances where subjective reports are weakly associated with objective measures of food intake as well as situations where the perceived urge to eat or the experienced taste of food is clearly linked to consumption.
The nature of subjective forms of "liking" and "wanting" is that they are consciously experienced and subject to interference from other thoughts and subjective states. Therefore, their relationship to human behavior is frequently contaminated. In addition, because it can be argued that not all behavior is under direct control of conscious events, these components can have an explicit and an implicit dimension (ie, one that is conscious and one that is covert). The influence of implicit processes of "liking" and "wanting" on behavior do not require conscious experience and may be more automated and related to behavior where subjective monitoring is low. The distinction between human subjective experience of "liking" and "wanting," and behavior influenced by implicit reward processes that operate outside awareness suggests it is necessary to delineate these processes. This means that they have much greater resolving potential for understanding the role of reward on eating [7]. Therefore, "liking" and "wanting" can be viewed as psychological components of reward operating at implicit (subconscious, automatic) and explicit (conscious, introspective) levels. As hypothetical constructs these entities are not directly observed; their logical status is that of intervening variables. They are an essential component of theory construction because they have high explanatory value and they help to organize thinking about the causes of behavior (an alternative approach is to simply have an inventory of behavioral acts). Therefore, in developing the theory of hedonic food processes the logical status of the concepts is important. This has implications; first it is important to demonstrate that the processes are separate or dissociable (to determine that two processes rather than one are needed), and second to validate the processes by relating them to measurable quantifiable operations. In this way the constructs become anchored to agreed observable entities.

\section{Measuring 'Liking' and 'Wanting' in Humans}

The translation of "liking" and "wanting" into measureable, behavioral operations is not without its challenges. Successful procedures must encompass the ability to not only reflect the existence of the different components of reward, but also prevent confounding one component with another to allow for dissociations to be detected. Explicit measures of food "liking" and "wanting" most commonly use psychometric techniques such as numerical scales and visual analogue scales. Questions such as "How pleasant would it be to taste some of this food now?" and "How pleasant is the taste of this food?" are often used to measure explicit "liking" for food, whereas questions such as "How strong is your desire to eat this food?" and "How much do you want this food?" are often used for the assessment of explicit "wanting." These techniques are limited by the accuracy of self-reporting and methodologic issues such as "end avoidance" and social desirability. However, if used 
carefully they can be quite sensitive to even subtle experimental manipulations and they frequently predict ingestive behavior. Although people tend to be very good at estimating and reporting their explicit "liking" for food, they are often unable to accurately gauge their implicit "wanting" for food. Implicit "wanting" concerns the core motivational aspects of rewardseeking behavior. Therefore, measures that reflect motivational responses to food and related cues can be said to contain at least an element of implicit "wanting." The more spontaneous the response, the closer that behavior is likely to reflect the core process of "wanting" without contamination from subjective processes. Importantly, implicit "wanting" may not be adequately captured by the nonspecific desire for food in general. "Wanting" implies a target with a direction, not just a force.

In recent years a range of techniques have been adapted or developed to assess more implicit forms of "wanting." These methods tend to involve tasks that require an instrumental response such as a button press or mouse click in relation to the simulated or actual presence of food or food cues. Techniques tend to fall into one of two categories. The first type depends on the subjects' willingness to expend effort to obtain a target food - usually something highly palatable and suited to the subject's personal preference. These measures operationalize "wanting" as the reinforcing value of the food or how hard an individual is willing to work to gain access to food compared with an alternative reward [18]. The second type of technique depends on the compatibility of a food or food cue with a time-critical approach-related response. These techniques such as the Stroop, Visual Probe, and Stimulus-response compatibility tasks measure reaction times after exposure to a food compared with a control or alternative food category [19]. The resulting "approach bias," affected by the attention grabbing/maintaining properties of the food and reflected in the speed of the response, is interpreted as a measure of motivational value or "wanting" [20, 21].

\section{The Role of 'Liking' and 'Wanting' in Disordered Eating}

It is reasonable to suggest that in instances where processes of "liking" and "wanting" dissociate or come to malfunction in some way (become either enhanced or attenuated), the resulting impact on behavior may resemble certain forms of disordered eating [22]. Such dissociations in the hedonic response to food may help to characterize particular phenotypes that are susceptible to overeating.

\section{Obesity and Weight Gain}

Previous research suggests that overweight and obese individuals have higher food "wanting" than normal weight individuals. Obese subjects have been shown to work harder to obtain snack food compared with engaging in alternative activities such as reading, playing computer games, or access to fruit or vegetable snack alternatives [23, 24, 25•]. In these studies the increased "wanting" was observed alongside equivalent or decreased "liking" for the target foods compared with nonobese persons. In children, self-reported willingness to expend effort in exchange for quantities of candy compared with a non-food reward was predictive of weight gain after 1 year [26]. Increased food "wanting" in overweight and obese individuals has also been demonstrated using other measurement paradigms. For example, measures of attentional bias, in which the inherent "attention-grabbing" properties of highenergy, palatable food are assessed against low-energy or non-food alternatives. Nijs et al. [27] compared overweight and obese females with normal weight controls using a visual probe task in conjunction with event-related potentials from electroencephalography (EEG) as a measure of attention allocation and found a trend for obese relative to lean individuals to orient more quickly to food cues. More recently, attentional bias for appetizing compared with unappetizing or non-food images was assessed in conjunction with brain imaging using functional magnetic resonance imaging (fMRI) in young females ranging from lean to obese [28•]. It was found that body mass index (BMI) was positively associated with attentional bias for food (appetizing or unappetising) compared with non-food stimuli; and during initial orientation to food, BMI also correlated with neural activation in the gustatory insula and frontal operculum regions. Furthermore, the authors conducted longitudinal analyses of change in BMI after 1 year and found that activation in the orbitofrontal cortex during initial orientation to appetizing food correlated with increased BMI. From the evidence it would appear obesity is associated with an increased "wanting" for food.

\section{Binge and Compulsive Eating}

Binge eating behavior is often associated with increased cravings for sweet foods [29, 30] and increased consumption of food in ad libitum eating tasks [31, 32] accompanied by a perceived loss of control over eating, and guilt following a binge [33]. Recently, researchers have focused on binge eating as a relevant "hedonic phenotype" of overconsumption and obesity [34-37, 38••, 39]. Svaldi et al. [39] examined attentional allocation in overweight females with and without binge eating disorder (BED) in EEG responses to high- and lowenergy foods. They found that subjects with BED had increased electrophysiologic activity in response to highenergy food images, consistent with greater food "wanting." Another study comparing obese individuals with and without BED examined genetic and psychological markers of hedonic eating [38••]. In addition to a psychometric assessment of 
"hedonic hunger" [40], polymorphisms of the DRD2 (TaqIA) and OPRM1 $(A 118 G)$ genes were examined as potential markers of dysregulated "liking" and "wanting." It was found that the obese BED group scored higher on the hedonic eating measure, and also had a higher frequency of the A2 allele of TaqIA and $\mathrm{G}$ allele of $A 118 \mathrm{G}$ variants compared with obese controls. Because the A2 allele is associated with a higher density of DRD2 receptors in the striatum, and the $G$ allele is associated with greater opioid function, the authors suggested that binge eating is a specific phenotype of obesity potentially characterized by distinct elevations in "liking" and "wanting" [38*0]. Although more commonly studied in the overweight and obese, evidence suggests that binge eating behaviors also occur in healthy nonobese adults who experience similar adverse psychological and behavioral outcomes [41•]. Using a psychometric assessment of binge eating severity [42], Finlayson et al. [41•] found that high scorers had an enhanced "liking" for all foods assessed but they had increased "wanting" specific for high-fat sweet foods only. This increased "wanting" was demonstrated by subjects responding faster to images of high-fat sweet foods, suggesting that these foods had an enhanced motivational value compared with the other food categories. Furthermore, the enhanced "wanting" for sweet foods in high binge scores coincided with them consuming 50\% more high-fat sweet foods in an ad libitum test meal. These data suggest that the tendency to binge eat is characterized by a heightened "wanting" and "liking" for food, and may form part of a "hedonic phenotype" for overconsumption, even in pre-obese individuals.

\section{Restrained Eating}

Individuals defined as restrained eaters [43] are typified by their intent to restrict their caloric intake, with a specific emphasis on the restriction of unhealthy foods. However, restrained eaters are often unsuccessful in restricting their intake [44-46]. One theory of how restrained eating paradoxically leads to overconsumption is Goal Conflict Theory [47]. Goal Conflict Theory suggests that restrained eaters hold two conflicting goals with regard to food: the goal of eating palatable foods; and the goal of controlling their weight (through caloric restriction). The theory states that the goal to restrict food intake is predominant but can be inhibited by the automatic activation of the goal to eat palatable food when confronted with a cue to eat. Experimental examination of this increased sensitivity to the hedonic properties of food suggests that it is attributable to an increase in "wanting" as opposed to "liking" for food. A study by Hoefling and Strack [48] examined restrained and unrestrained eaters' "liking" and "wanting" for high- and low-calorie food cues. They found that restrained eaters had a decreased "liking" for food, but showed an increased "wanting" for them when compared with unrestrained eaters. More recently, Veenstra and de Jong [49] demonstrated that although restrained and unrestrained eaters had a similar "liking" for food, restrained eaters showed enhanced "wanting." These findings suggest that food "wanting" may be more important in determining the success of restrained eaters and emphasize the importance of structuring eating patterns and limiting exposure to stimulation from food in the environment.

\section{Are 'Liking' and 'Wanting' Involved in 'Food Addiction'?}

"Food addiction" has recently been proposed as a valid phenotype of obesity [50॰]. The theoretical basis for the existence of food addiction in humans is mostly drawn from clinical observations, the scientific literature on drugs of abuse, and in experimental animal models [51-54]. It has been suggested that chronic exposure to the combination of high palatability and high-energy-dense foods can alter central neurotransmitter systems causing susceptible individuals to display symptoms of addictive behavior which, in turn, can lead to further overeating and weight gain. Davis and Carter [52] proposed that obese individuals with BED should be considered as having a form of "food addiction" and they draw parallels between the disorder and behaviors associated with drugs of abuse. They argue that like drug addicts, individuals with BED experience a persistent desire and a loss of control with regard to the consumption of palatable foods and continue to consume it despite the negative outcomes (eg, feelings of guilt and an increase in body weight). More recently, they presented evidence using a newly developed food addiction scale, the Yale Food Addiction Scale (YFAS), which aims to identify individuals with addictive tendencies toward food [50•]. Using a sample of overweight adults they found that $76 \%$ of the group who met the criteria for "food addiction" measured by the YFAS were also comorbid for BED, more impulsive, had more addictive personality traits, and reported an increased number of food cravings [50 $]$.

At the present time the human evidence on this issue arises almost exclusively from research conducted in North America, and from authors and researchers embedded in this sociocultural landscape. The food environment in North America is highly specialized to promote excessive consumption; however, this food is contained within a socioeconomic system that encourages purchasing and consumption of many items (not only food) without limits. Therefore, it may be premature to regard addiction to foods as a universal phenomenon that has global penetration. At the moment it is not possible to detect whether or not the excessive consumption of certain foods in the North American food supply arises solely from the orosensory properties of these foods or from the way in which this overconsumption is legitimized and 
promoted by prevailing values in the surrounding culture [55]. Furthermore, considering that obesity is a highly heterogeneous condition with numerous causal factors and mechanisms, the term "food addict," when used to define the eating behavior of many millions of individuals may be unhelpful, and might only be relevant to a much smaller number of people whose behavior resembles that of substance dependence, such as in the case of individuals with severe BED [52].

Whether or not the term addiction should be applied, it can certainly be recognized that the hedonic response to food is a psychological and biological reality and that the pleasure derived from eating forms an important part of the quality of life for many human beings in whichever culture they live in. Orosensory qualities of food can only engender an affective response if brain processes exist to mediate their physical impact on the subjective state. The hedonic "hotspots" and dopamine pathways within this circuitry have been regarded as providing a neural basis for processes of "liking" and "wanting" [11]. This approach has facilitated understanding of how the hedonic system may function physiologically and has added sensitivity to the experimental study of hedonically driven eating. The terms "liking" and "wanting" can be dissociated semantically (although they are often used as synonyms in common speech) and this gives further substance to the separation of the processes through experimental analysis and observational scrutiny. The validity of such a distinction can be demonstrated through the experimental dissociation of the functional effects ascribed to food "liking" and "wanting" [7, 56, 57]. The separate identities for "liking" and "wanting" provide increasing analytical power to explore the complexities of the effects of food hedonics on eating. The further distinction (experimentally validated) between explicit and implicit processes adds theoretical justification to commonly observed self-statements about appetite ("I've just got to have that food; I don't know why but I have to"; "implicit wanting"). We propose that these separate processes (that we refer to as "liking" and "wanting") can exert separate (and sometimes conjoint) effects on eating and therefore represent risk factors for overconsumption and some forms of disordered eating.

\section{Conclusions}

The hedonics of food consumption involve components of food "liking" and "wanting" that operate within a psychobiological system of appetite control. These processes have their neural correlates in animals (and humans) and progress has been made in distinguishing and measuring the behavioral expression of these processes in humans. Several studies implicate enhanced "wanting" in the behavior of those who are obese or at risk of gaining weight; however, enhanced "wanting" in conjunction with greater "liking" for food appear to underpin the tendency to binge eat in obese and pre-obese individuals. Therefore, instances where processes of "liking" and "wanting" become either enhanced or dissociated can be observed in certain forms of disordered eating. Identification of malfunctions in the hedonic response to food may help to characterize particular phenotypes that are susceptible to overeating and inform the optimization of weight control strategies by targeting food "liking" and/or "wanting" to help restore homeostatic controls over food intake.

Disclosure No potential conflicts of interest relevant to this article were reported.

\section{References}

Papers of particular interest, published recently, have been highlighted as:

- Of importance

•. Of major importance

1. Finucane MM, Stevens GA, Cowan MJ, et al. National, regional, and global trends in body-mass index since 1980: systematic analysis of health examination surveys and epidemiological studies with 960 country-years and $9 \cdot 1$ million participants. Lancet. 2011;377(9765):557-67.

2. Young LR, Nestle M. The contribution of expanding portion sizes to the U.S. obesity epidemic. Am J Public Health. 2002;92:246-9.

3. Tremblay MS, Willms JD. Is the Canadian childhood obesity epidemic related to physical inactivity? Int J Obes Relat Metab Disord. 2003;27(9):1100-5.

4. Lutter M, Nestler EJ. Homeostatic and Hedonic Signals Interact in the Regulation of Food Intake. J Nutr. 2009;139(3):629-32.

5. Blundell JE, Finlayson G. Is susceptibility to weight gain characterized by homeostatic or hedonic risk factors for overconsumption? Physiol Behav. 2004;82(1):21-5.

6. Blundell JE. Perspective on the Central Control of Appetite. Obesity. 2006;14(7S):S160-3.

7. Finlayson G, King N, Blundell JE. Liking vs. wanting food: Importance for human appetite control and weight regulation. Neurosci Biobehav Rev. 2007;31(7):987-1002.

8. Campfield LA, Smith FJ, Guisez Y, et al. Recombinant mouse OB protein: evidence for a peripheral signal linking adiposity and central neural networks. Science. 1995;269:546-9.

9. Berridge KC. Food reward: brain substrates of liking and wanting. Neurosci Biobehav Rev. 1996;20:1-25.

10. Berridge KC. The debate over dopamine's role in reward: the case for incentive salience. Psychopharmacology. 2007;191(3): 391-431.

11. Pecina S, Smith KS, Berridge KC. Hedonic hot spots in the brain. Neuroscientist. 2006;12(6):500-11.

12. Berridge KC. Pleasures of the brain. Brain Cogn. 2003;52(1): 106-28.

13. •• Berridge, KC, Ho, C-Y, Richard, JM, \& DiFeliceantonio, AG: The tempted brain eats: pleasure and desire circuits in obesity and eating disorders. Brain Res 2010, 1350:43-64. This article reviews evidence, and draws conclusions about the underlying brain mechanisms of "liking" and "wanting" and their role in obesity and eating disorders, including food addiction. Additionally, interactions between brain reward circuitry and regulatory mechanisms of hunger and satiety are discussed. 
14. Kelley AE, Baldo BA, Pratt WE. A proposed hypothalamicthalamic-striatal axis for the integration of energy balance, arousal, and food reward. J Comp Neurol. 2005;493(1):72-85.

15. Smith KS, Berridge KC, Aldridge JW. Disentangling pleasure from incentive salience and learning signals in brain reward circuitry. PNAS. 2011;108(27):E255-64.

16. Berridge $\mathrm{KC}$. Motivation concepts in behavioral neuroscience. Physiol Behav. 2004;81:179-209.

17. Simpson JA, Weiner ESC. The Oxford English dictionary. 2nd ed. Oxford: Clarendon; 1989.

18. Epstein LH, Leddy JJ, Temple JL, Faith MS. Food reinforcement and eating: a multilevel analysis. Psychol Bull. 2007;133 (5):884-906

19. Nathan, PJ, O’Neill, BV, Mogg, K, Bradley, BP, Beaver, J, Bani, M, Merlo-Pich, E, Fletcher, PC, Swirski, B, Koch, A, Dodds, CM, \& Bullmore, ET: The effects of the dopamine D3 receptor antagonist GSK598809 on attentional bias to palatable food cues in overweight and obese subjects. International J Neuropsychopharm 2011, FirstView, 1-13.

20. Brignell C, Griffiths T, Bradley BP, Mogg K. Attentional and approach biases for pictorial food cues. Influence of external eating. Appetite. 2009;52(2):299-306.

21. Finlayson G, King N, Blundell J. The role of implicit wanting in relation to explicit liking and wanting for food: implications for appetite control. Appetite. 2008;50:120-7.

22. Berridge KC. 'Liking' and 'wanting' food rewards: brain substrates and roles in eating disorders. Physiol Behav. 2009;97 (5):537-50.

23. Saelens BE, Epstein LH. Reinforcing value of food in obese and non-obese women. Appetite. 1996;27:41-50.

24. Epstein LH, Temple JL, Neaderhiser BJ, et al. Food reinforcement, the dopamine D2 receptor genotype, and energy intake in obese and nonobese humans. Behav Neurosci. 2007;121(5):877-86.

25. • Giesen, JCAH, Havermans, RC, Douven, A, et al.: Will Work for Snack Food: The Association of BMI and Snack Reinforcement. Obesity 2010, 18(5):966-970. Using the relative reinforcing value of food paradigm, the authors showed that overweight/obese individuals will work harder for access to snack foods compared with normal weight controls.

26. Hill C, Saxton J, Webber L, et al. The relative reinforcing value of food predicts weight gain in a longitudinal study of 7-10-y-old children. Am J Clin Nutr. 2009;90(2):276-81.

27. Nijs IMT, Muris P, Euser AS, Franken IHA. Differences in attention to food and food intake between overweight/obese and normalweight females under conditions of hunger and satiety. Appetite. 2010;54(2):243-54.

28. • Yokum, S, Ng, J, \& Stice, E.: Attentional Bias to Food Images Associated with Elevated Weight and Future Weight Gain: An fMRI Study. Obesity 2011, 19(9):1775-1783. In this fMRI study, greater lateral orbitofrontal cortex activation (indicative of an elevated reward circuitry responsiveness) during initial orientation of attention to appetizing food cues predicted future increases in BMI in a sample of adolescent females.

29. Greeno CG, Wing RR, Shiffman S. Binge antecedents in obese women with and without binge eating disorder. J Consult Clin Psychol. 2000;68(1):95-102.

30. Kampov-Polevoy AB, Alterman A, Khalitov E, Garbutt JC. Sweet preference predicts mood altering effect of and impaired control over eating sweet foods. Eat Behav. 2006;7(3):181-7.

31. Geliebter A, Hassid G, Hashim SA. Test meal intake in obese binge eaters in relation to mood and gender. Int J Eating Disord. 2001;29:488-94.

32. Latner JD, Rosewall JK, Chisholm AM. Food volume effects on intake and appetite in women with binge-eating disorder and weight-matched controls. Int J Eating Disord. 2009;42 (1):68-75
33. Ricca V, Castellini G, Losauro C, et al. Correlations between binge eating and emotional eating in a sample of overweight subjects. Appetite. 2009;53(3):418-21.

34. Nathan PJ, Bullmore ET. From taste hedonics to motivational drive: central mu-opioid receptors and binge-eating behaviour. Int J Neuropsychopharmacol. 2009;12(07):995-1008.

35. Nathan, PJ, O’Neill, BV, Bush, MA, et al.: Opioid Receptor Modulation of Hedonic Taste Preference and Food Intake: A Single-Dose Safety, Pharmacokinetic, and Pharmacodynamic Investigation With GSK1521498, a Novel \{micro\}-Opioid Receptor Inverse Agonist. J Clin Pharmacol 2011.

36. Davis C, Levitan RD, Kaplan AS. Dopamine transporter gene (DAT1) associated with appetite suppression to methylphenidate in a case-control study of binge eating disorder. Neuropsychopharmacology. 2007;32:2199-206.

37. Davis C, Levitan RD, Kaplan AS, et al. Reward sensitivity and the D2 dopamine receptor gene: A case-control study of binge eating disorder. Prog Neuropsychopharmacol Biol Psychiatry. 2008;32 (3):620-8.

38. - Davis CA, Levitan RD, Reid C, et al. Dopamine for "wanting" and opioids for "liking": a comparison of obese adults with and without binge eating. Obesity 2009, 17(6):1220-5. Examining genetic (Taq1A and OPRM1 polymorphisms) and psychological (hedonic hunger) risk factors for overconsumption, the authors found that obese adults with BED were more likely to have a enhanced hedonic genotype (G+ and A1-) and greater hedonic hunger than obese individuals without BED, indicating that the propensity to binge eat may be impacted by elevations in "liking" and "wanting".

39. Svaldi J, Tuschen-Caffier B, Peyk P, Blechert J. Information processing of food pictures in binge eating disorder. Appetite. 2010; 55(3):685-94

40. Lowe MR, Butryn ML. Hedonic hunger: A new dimension of appetite? Physiol Behav. 2007;91(4):432-9.

41. - Finlayson, G, Arlotti, A, Dalton, M, et al.: Implicit wanting and explicit liking are markers for trait binge eating. A susceptible phenotype for overeating. Appetite 2011, In press. The investigators demonstrate that binge eating severity is characterized by enhanced "liking" and implicit "wanting" for food in normal weight females highlighting the relevance of examining disordered eating in the "pre-obese."

42. Gormally J, Black S, Daston S, Rardin D. The assessment of binge eating severity among obese persons. Addict Behav. 1982;7: $47-55$.

43. Herman, CP, \& Polivy, J: Restrained eating. In A. J. Stunkard (Ed), Obesity Philadelphia: Saunders; 1980:208-225.

44. Fedoroff IC, Polivy J, Herman CP. The effect of pre-exposure to food cues on the eating behavior of restrained and unrestrained eaters. Appetite. 1997;28:33-47.

45. Herman CP, Mack D. Restrained and unrestrained eating. J Pers. $1975 ; 43: 647-60$

46. Jansen A, Van den Hout MA. On being led into temptation: "Counterregulation" of dieters after smelling a "preload". Addict Behav. 1991;16:247-53.

47. Stroebe W, Papies EK, Aarts H. From Homeostatic to Hedonic Theories of Eating: Self-Regulatory Failure in Food-Rich Environments. Appl Psychol. 2008;57:172-93.

48. Hoefling A, Strack F. The tempting effect of forbidden foods. High calorie content evokes conflicting implicit and explicit evaluations in restrained eaters. Appetite. 2008;51(3):681-9.

49. Veenstra EM, de Jong PJ. Restrained eaters show enhanced automatic approach tendencies towards food. Appetite. 2010; $55: 30-6$.

50. - Davis, C, Curtis, C, Levitan, RD, et al. Evidence that 'food addiction' is a valid phenotype of obesity. Appetite 2011, 57:711-717. The authors add to the debate on "food addiction" 
being a phenotype of obesity, presenting data that suggests individuals who meet the criteria for "food addiction" (using the newly developed YFAS) have a greater comorbidity with BED compared with age- and weight-matched controls.

51. Gold MS, Frost-Pineda K, Jacobs WS. Overeating, binge eating and eating disorders as addictions. Psychiatr Ann. 2003;33(2): 117-22.

52. Davis C, Carter JC. Compulsive overeating as an addiction disorder. A review of theory and evidence. Appetite. 2009;53(1): $1-8$.

53. Pelchat ML. Food Addiction in Humans. J Nutr. 2009;139(3): $620-2$.
54. Avena NM, Rada P, Hoebel BG. Sugar and Fat Bingeing Have Notable Differences in Addictive-like Behavior. J Nutr. 2009;139 (3):623-8.

55. Kessler D. The end of overeating: taking control of the insatiable American appetite. New York: Rodale Books Inc; 2009.

56. Griffioen-Roose S, Finlayson G, Mars M, et al. Measuring food reward and the transfer effect of sensory specific satiety. Appetite. 2010;55(3):648-55.

57. Verschoor E, Finlayson G, Blundell J, et al. Effects of an acute alpha-lactalbumin manipulation on mood and food hedonics in high- and low-trait anxiety individuals. Br J Nutr. 2010;104 (4):595-602. 